\title{
Del macro al microcarcinoma tiroideo: cambios en las características de presentación del cáncer de tiroides en un centro universitario chileno en 20 años
}

\author{
LORENA MOSSO ${ }^{1}$, CLAUDIA CAMPUSANO ${ }^{1}$, \\ HERNÁN GONZÁLEZ², JOSÉ MIGUEL DOMÍNGUEZ ${ }^{1}$, PATRICIO SALMAN', \\ VALERIA SUAZO ${ }^{1}$, ANTONIETA SOLAR ${ }^{3}$, JAIME CERDA ${ }^{4}$
}

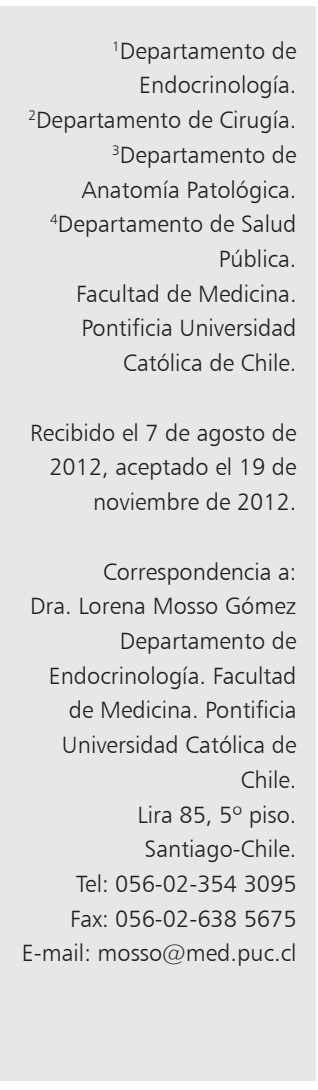

\section{From macro to micro thyroid carcinoma: records of a clinical hospital from 1991 to 2010}

Background: The prevalence of thyroid cancer has increased, particularly in nodules smaller than $10 \mathrm{~mm}$, probably due to the growing use of routine thyroid ultrasound. There is controversy about the biological behavior of microcarcinomas and the relevance of their early detection. Aim: To characterize the clinical presentation of thyroid cancer over 20 years in an University medical center and to evaluate the differences between macro and microcarcinomas. Patients and Methods: We reviewed 1547 surgical biopsy records of thyroid cancer in our institution obtained between 1991 and 2010. Results: We observed a sustained increase in the rate of thyroidectomies for thyroid cancer (per 1000 surgical procedures) in the study period. Papillary, follicular, mixed, medullary and anaplastic carcinomas were observed in 95, 3, 2, 0.5 and $0.1 \%$ of biopsies, respectively. The incidence of tumors of less than $10 \mathrm{~mm}$ (microcarcinoma) also increased. Those findings were associated with a significant decrease in tumor aggressiveness, determined by a low frequency of surgical margin involvement of thyroid capsule, perithyroid tissue invasion, vascular permeation and lymph node metastases. Conclusions: The increased prevalence of thyroid cancer, especially of microcarcinomas, may reflect the greater use of diagnostic ultrasound or represent a real change in the biological behavior of this disease and our data suggest that further studies are needed to know the impact of early treatment in the outcome of those patients because of the real less histologyc agressiveness of microcarcinomas.

(Rev Med Chile 2013; 141: 442-448).

Key words: Papillary thyroid microcarcinoma; Thyroid neoplasms; Thyroid nodule.
1 cáncer de tiroides, y específicamente el carcinoma papilar de tiroides, es la neoplasia maligna endocrina más frecuente y su incidencia ha ido en aumento exponencial en los últimos años. En diferentes series da cuenta de $0,85-2,5 \%$ de incidencia y $0,21-0,3 \%$ de las muertes por cáncer ${ }^{1}$.

El aumento de casos ha ido en paralelo a la aparición de nuevas técnicas de tamizaje y al uso masivo de la ecografía ${ }^{2}$.

Clásicamente se ha considerado como un tumor de buen pronóstico cuando es detectado y tratado en etapas precoces, teniendo una mortalidad global inferior al 15\% a 20 años, siendo menor aun en los carcinomas papilares ${ }^{1}$.

El carcinoma papilar de tiroides representa 75- 
$85 \%$ de todos los casos, seguido del folicular, con alrededor de $10-15 \%$, medular 1-3\% y anaplástico con menos de $1 \%{ }^{3}$.

Dentro de la práctica endocrinológica habitual en nuestro país, existe la percepción de un incremento de los casos de cáncer papilar de tiroides durante los últimos años, pero no existen registros nacionales que avalen con exactitud esta afirmación.

El aumento de casos de cáncer operados, no necesariamente refleja un aumento de la incidencia real de la enfermedad y este aumento podría estar dado por cualquiera de los siguientes factores: precocidad del diagnóstico clínico, mayor utilización de tecnologías diagnósticas (ecografía, punción tiroidea), mejor estudio de piezas quirúrgicas o un real cambio en el comportamiento biológico de esta patología.

Un real aumento en la incidencia de estos tumores ha sido corroborado por trabajos extranjeros en que se ha confirmado un mayor número de casos en las últimas 2 décadas. En Francia se ha comunicado un dramático aumento de la incidencia de cáncer tiroideo llegando a 8,1\% y $6,2 \%$ por año, en mujeres y hombres respectivamente ${ }^{4}$. En Estados Unidos de Norteamérica se comunicó un aumento de incidencia de 2,4 veces (3,6 a 8,7 por 100.000 habitantes) entre 1973 y $2002^{5}$. El aumento de prevalencia ha sido también reportado por varios otros países como Canadá, Italia, Alemania, España y Australia, entre otros ${ }^{6,7}$. En todas las casuísticas presentadas, este hallazgo se debe principalmente a un mayor número de carcinomas papilares, no apreciándose aumento de los carcinomas foliculares o medulares.

Los registros nacionales no siempre representan fielmente el comportamiento de las patologías oncológicas y la realidad de diferentes regiones de Chile no es necesariamente homogénea. En nuestro país no contamos con un registro nacional de cáncer de tiroides. Un dato comunicado por nuestro grupo, muestra que existe un aumento real de la incidencia de cáncer tiroideo mostrado por el aumento de la tasa de egresos hospitalarios por esta patología, sin embargo, no conocemos las características de estos tumores ${ }^{8,9}$.

El objetivo de este trabajo es describir los hallazgos demográficos y anatomopatológicos de los cánceres de tiroides operados en la Pontifica Universidad Católica de Chile, en los últimos 20 años.

\section{Material y Métodos}

Se analizaron todos los registros de biopsias quirúrgicas de tiroidectomías realizadas en el Hospital Clínico de la Pontificia Universidad Católica de Chile (HCPUC) entre enero de 1991 y diciembre de 2010 y que tuvieran el diagnóstico final de cáncer de tiroides.

Se registró la edad al diagnóstico y género de los pacientes. Se especificaron el diámetro tumoral mayor, tipo histológico, presencia de compromiso de la cápsula tumoral, del borde quirúrgico, del tejido peritiroideo, permeación vascular y compromiso ganglionar, así como la existencia de uni o multifocalidad. Según el diámetro mayor tumoral, se clasificaron como microcarcinomas, los cánceres menores a $10 \mathrm{~mm}$. Los datos fueron obtenidos de los informes de biopsia hechos por distintos patólogos miembros del Departamento de Anatomía Patológica con técnica hematoxilinaeosina durante los años mencionados; no fueron revisados ni modificados por otros patólogos.

Las variables numéricas se presentan como promedio \pm desviación estándar y las categóricas como número de casos y porcentajes. Para estudiar la asociación entre variables categóricas se usó el test de $\chi^{2}$. La evolución de la incidencia de cáncer de tiroides durante el período señalado se estudió mediante correlación de Pearson o Spearman entre el año de diagnóstico y la incidencia de cáncer en cada año, dependiendo si las variables tenían distribución normal o no, respectivamente. Se consideró significativo un valor $\mathrm{p}<0,05$. Para el análisis estadístico se utilizó el programa SPSS para Windows versión 15.0 (SPSS Inc, Chicago).

Este trabajo fue aprobado por el comité de ética de nuestro hospital.

\section{Resultados}

En el período de estudio, 1.547 pacientes fueron tiroidectomizados con diagnóstico final de cáncer de tiroides. Se observó un aumento progresivo de la tasa de tiroidectomías por cáncer tiroideo por cada 1.000 cirugías/año como se muestra en la Figura 1 (rho Spearman 0,961; p < 0,001). Los tipos histológicos de cáncer tiroideo se distribuyeron de la siguiente forma: carcinoma papilar $1.464(94,6 \%)$, carcinoma folicular $40(2,6 \%)$, carcinoma medular $30(1,9 \%)$, carcinoma mixto 

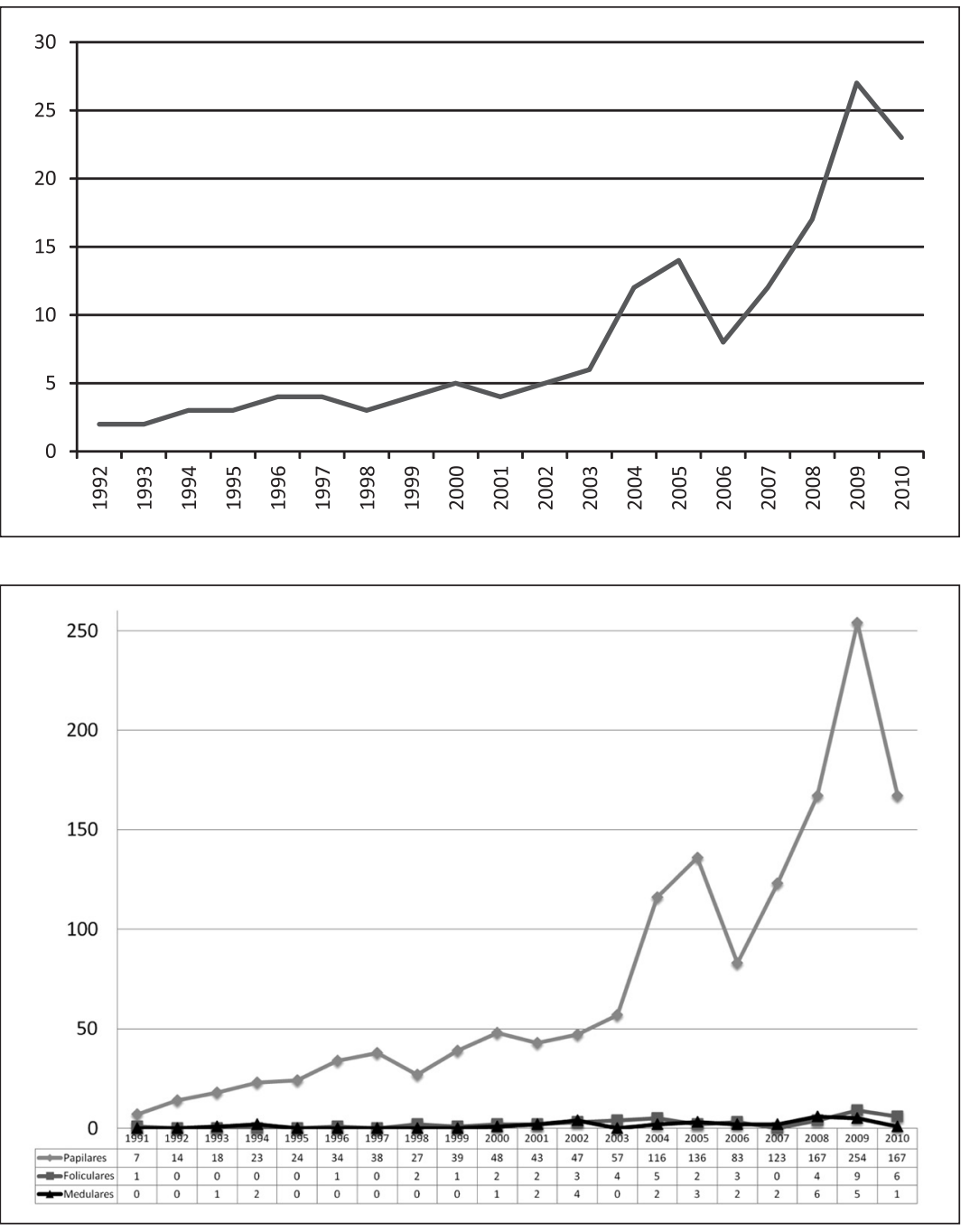

Figura 1. Tasa de tiroidectomías por cáncer de tiroides vs cirugías totales en nuestro centro por año.

Tabla 1 Sexo y edad según tipo histológico del cáncer de tiroides

\begin{tabular}{|lccc|}
\hline & $\begin{array}{c}\text { Papilar } \\
\mathbf{n = 1 . 4 6 4}\end{array}$ & $\begin{array}{c}\text { Folicular } \\
\mathbf{n = 4 0}\end{array}$ & $\begin{array}{c}\text { Medular } \\
\mathbf{n}=\mathbf{3 0}\end{array}$ \\
\hline Edad (promedio $\pm \mathrm{DE}$ ) rango & $43,7 \pm 14,2$ & $53,6 \pm 18,5$ & $44,0 \pm 17,9$ \\
Mujeres & $1.240(84,7 \%)$ & $32(80,0 \%)$ & $24(80.0 \%)$ \\
\hline
\end{tabular}

papilar y folicular $5(0,4 \%)$, carcinoma anaplástico $1(0,1 \%)$ y otros tipos $5(0,4 \%)$. En la Figura 2 se muestra el número de casos operados por año de acuerdo a histología, si bien existe un aumento de todos los tipos de cáncer esto es más significativo para el cáncer papilar. Al comparar los distintos tipos histológicos no hubo diferencia en la distribución por sexo y edad (Tabla 1).
El carcinoma papilar fue el subtipo más frecuente en esta serie, la edad promedio para estos pacientes fue de 43,7 $\pm 14,2$ años y la mediana 43,0 (rango intercuartil = 19 años), la distribución de la variable edad mostró un patrón normal que se muestra en Figura 3 y 1.240 (84,7\%) fueron mujeres.

El 42,3\% del total de cánceres tenían un diáme- 

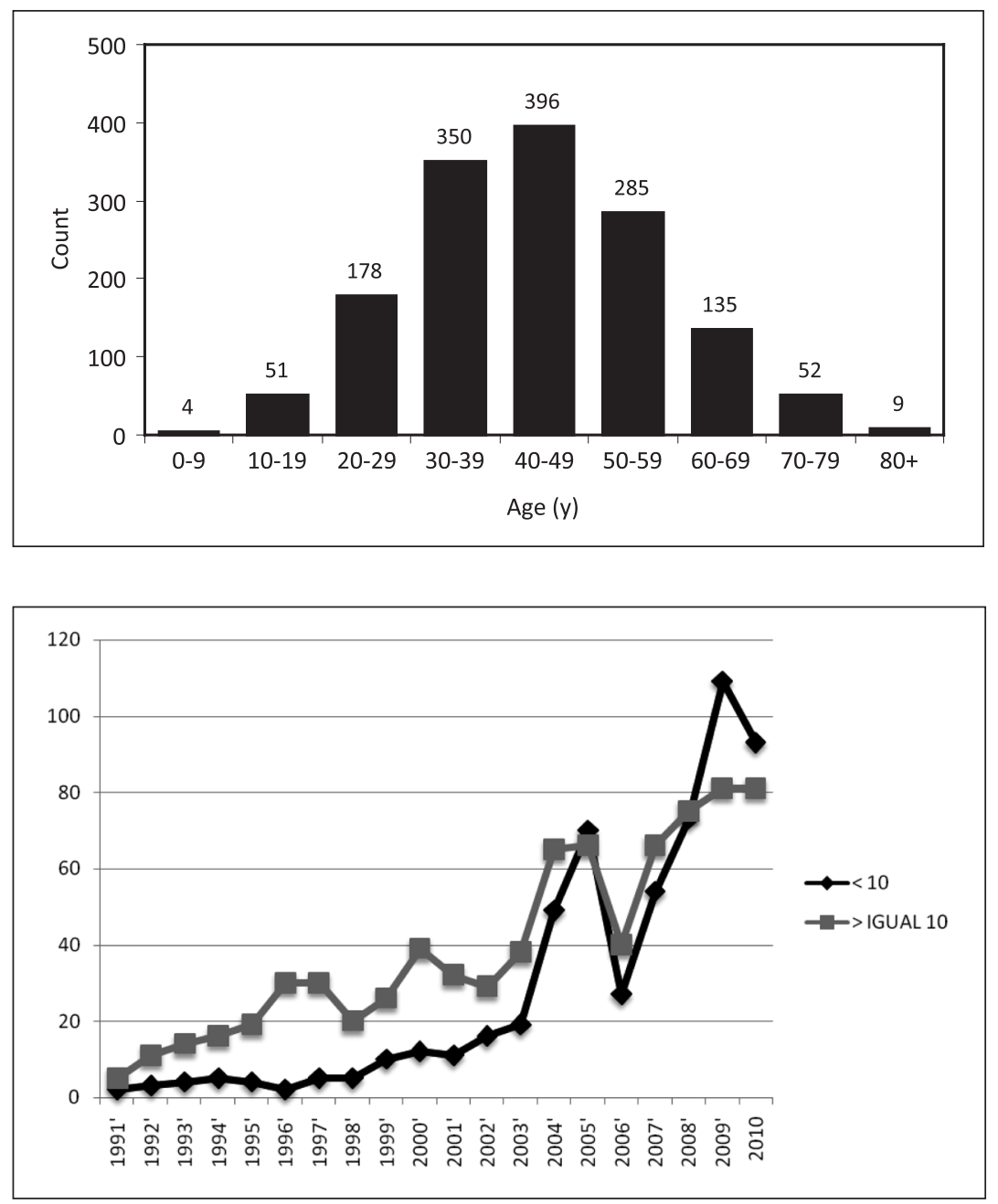

Figura 3. Número de casos distribuidos por edad de pacientes con cáncer papilar $\mathrm{n}=1.464$.
Figura 4. Distribución de casos según tamaño mayor o menor de $1 \mathrm{~cm}$. tro mayor inferior a $10 \mathrm{~mm}$ (microcarcinomas). Se observó un aumento progresivo de la prevalencia en el tiempo de macro y microcarcinomas (correlación de Pearson: $\mathrm{r}+0,891, \mathrm{p}<0,001)$. En 1991 el porcentaje de microcarcinomas era 14,3\%, y en 2010 era 55,7\%, llegando en este año a ser más frecuentes que los tumores de $10 \mathrm{~mm}$ o más de diámetro mayor (Figura 4). En la evolución se observa un aumento sostenido de la proporción de microcarcinomas, y en los últimos 2 años son la forma predominante, con respecto a los tumores de $10 \mathrm{~mm}$ o más.

No hubo diferencia en la distribución de género entre micro y macrocarcinomas.

Entre las características histopatológicas analizadas, los microcarcinomas tuvieron menor riesgo de invasión de borde, invasión de cápsula, extensión a tejido peritiroideo, permeación vascular y compromiso ganglionar. En los macrocarcinomas existió un mayor número de casos de multifocalidad (Tabla 2).

\section{Discusión}

La frecuencia de diagnóstico de cáncer de tiroides ha aumentado en el mundo en los últimos 20 años y nuestros datos son consistentes con este fenómeno. Este hecho ha sido tema de gran discusión y controversia, dado que los datos apuntan a un aumento en la incidencia en la población general no expuesta a un riesgo específico conoci- 
Tabla 2. Características de los micro y macrocarcinomas

\begin{tabular}{|lccc|}
\hline Variable & $\begin{array}{c}\text { Microcarcinoma } \\
\mathbf{n}(\mathbf{\%})\end{array}$ & $\begin{array}{c}\text { Macrocarcinoma } \\
\mathbf{n ~ ( \% )}\end{array}$ & OR (IC 95\%) \\
\hline Femenino & $537(87,6)$ & $691(82,7)$ & $1,48(1,09-2,02)$ \\
Masculino & $76(12,4)$ & $145(17,3)$ & $0,35(0,24-0,51)$ \\
\hline Compromiso borde & $45(7,6)$ & $156(18,8)$ & $0,39(0,31-0,50)$ \\
Invasión de cápsula & $153(26,3)$ & $389(47,7)$ & $0,39(0,30-0,52)$ \\
Invasión de tejido peritiroideo & $89(16,6)$ & $256(33,7)$ & $0,28(0,20-0,41)$ \\
\hline Permeación vascular & $45(7,6)$ & $182(22,5)$ & $0,22(0,16-0,30)$ \\
\hline Metástasis linfáticas & $76(22,7)$ & $279(57,3)$ & $1,21(0,98-1,49)$ \\
\hline Multifocalidad & $232(36,6 \%)$ & $376(41,2 \%)$ & 100 \\
\hline
\end{tabular}

$\mathrm{do}^{1-7}$. Lo anterior pudiera ser explicado por mayor oportunidad diagnóstica de esta enfermedad y/o un real aumento de su incidencia. La metodología empleada y los datos disponibles en este trabajo no permiten la real diferenciación entre ambas opciones.

En nuestra experiencia, el aumento de casos se asocia claramente a un mayor número de diagnóstico de tumores menores de $1 \mathrm{~cm}$, que son difícilmente encontrados por examen físico o síntomas ${ }^{3}$ por lo que podemos postular que efectivamente, se realizan más exámenes de pesquisa lo que conlleva una mayor oportunidad diagnóstica.

La mayor posibilidad de diagnóstico temprano, puede ser un reflejo del mayor número de médicos, mayor accesibilidad y rendimiento de métodos diagnósticos (ecografía tiroidea, PAF, estudio cito-histológico), así como de la disponibilidad de tiroidectomías. No contamos con datos que muestren el número de los procedimientos ecográficos solicitados como tamizaje asintomático, pero es evidente que en 20 años ha aumentado exponencialmente el número total de estos procedimientos, tanto por el aumento de disponibilidad como por la disminución de los costos de esta tecnología.

La tecnología disponible actualmente para el estudio de imágenes, tanto en la capacidad de discriminación del ultrasonido de alta resolución como en el aumento de disponibilidad de equipos a lo largo de nuestro país, ha llevado a examinar población teóricamente sana, en la que se ha encontrado patología nodular del tiroides. Las normas actuales de manejo del nódulo tiroideo obligan a completar el estudio en muchos de ellos con una biopsia con aguja fina ${ }^{3,10}$.
La observación del aumento absoluto y relativo de los microcarcinomas por sobre los tumores de $10 \mathrm{~mm}$ o más, sugiere un diagnóstico y cirugía más precoz en la historia natural de la enfermedad.

Los estudios clásicos en autopsia, mostraban que hasta en 30\% de las tiroides estudiadas dirigidamente presentan algún microfoco de carcinoma papilar ${ }^{11} \mathrm{y}$ es probable que los hallazgos actuales estén haciendo clínicamente evidente este hecho. En nódulos tiroideos incidentales la incidencia de cáncer es de $5 \%{ }^{12}$. Por otra parte, la prevalencia de cáncer oculto de tiroides varía entre 3,9 y 4,1\% en población de USA siendo casi exclusivamente carcinomas papilares $^{11,12}$.

Nuestros datos demuestran que los microcarcinomas presentan menos características de riesgo histológico que los macrocarcinomas, en cuanto a menor de compromiso de borde del nódulo, de la cápsula tiroidea, del tejido peritiroideo, menor frecuencia de permeación vascular y de compromiso ganglionar. Este hallazgo es de gran relevancia, puesto que evidencia la importancia de un diagnóstico precoz que posibilite encontrarnos con tumores menos agresivos y con menos metástasis al diagnóstico.

Con respecto a la biología del carcinoma papilar, sabemos que es un tumor de lento crecimiento, por otra parte, Davies L et al demuestran que la mortalidad ha permanecido estable en la población a pesar de un incremento real en la incidencia de la enfermedad ${ }^{5}$. Estos mismos autores apuntan a la llamativa disociación entre el aumento de tasas de carcinoma papilar en 5 por 10.000 habitantes en comparación al aumento de sólo 0,15 por 10.000 habitantes de cánceres tiroideos metastásicos. Una 
explicación a lo anterior podría ser que estamos diagnosticando los casos más precozmente con características de riesgo histológico más benignas al diagnóstico como se observa en los datos que presentamos. En nuestra casuística demostramos que en los casos de macrocarcinoma existe además de los mayores criterios de riesgo histológico, un mayor número de focos tumorales que en los microcarcinoma. Este hecho puede reflejar que un diagnóstico precoz evita que se manifieste una condición genética predisponente a cáncer en toda la tiroides de ese individuo o que existiera menos posibilidades de invasión intratiroidea.

El aumento en el número de casos de cáncer tiroideo puede ser también explicado por un aumento real de la incidencia. La causa posible de un fenómeno así nos hace analizar si nuestra población está expuesta a mayores factores de riesgo conocidos para el carcinoma de tiroides ${ }^{5,9}$. Algunos autores han especulado que esto podría estar causado por una mayor exposición a carcinógenos ambientales como radiación o a una alta o excesiva ingesta de yodo, hecho real en Chile ${ }^{14,15}$ pero no hay pruebas de ello ${ }^{5,16}$. Con respecto a la radiación, en la actualidad su uso terapéutico se reserva para un número reducido de patologías malignas (su uso en patologías benignas está en desuso), sin embargo, su uso con fines diagnósticos ha ido en aumento lo que pudiera asociarse a aumento del cáncer de tiroides en los próximos años, especialmente, en la población pediátrica expuesta; lo que debe alertar al equipo médico a tomar todas las medidas necesarias para reducir la exposición a radiación en niños ${ }^{16}$.

Otro elemento a considerar es que recientemente se ha observado que la presencia de tiroiditis de Hashimoto se asocia a un aumento de la incidencia de cáncer de tiroides con OR de 2,96 y que esto podría deberse a la activación de la vía de PI3K/AKT ${ }^{17}$. En nuestra población existe un alto reporte de enfermedad tiroidea funcional y autoinmune, mayor que en otros países, según los datos de la ENS 2009-2010 Chile, por lo que una hipótesis de asociación de enfermedad autoinmune y mayor riesgo de cáncer tiroideo es posible de plantear aunque nuestro estudio no permite un mayor análisis en este sentido ${ }^{18}$.

En conclusión, los resultados de nuestro trabajo son acordes a lo publicado mostrando un aumento de la incidencia de cáncer tiroideo, especialmente papilar y más específicamente un aumento de los microcarcinomas tiroideos. Además sugiere que los microcarcinomas se asocian a menor agresividad en relación a los macrocarcinomas, lo cual es concordante con la menor mortalidad demostrada en el largo plazo cuando se operan pacientes con tumores de menos de $1 \mathrm{~cm}$ versus los de más de 1 $\mathrm{cm}^{19}$. A la luz de nuestros datos, es planteable que un diagnóstico precoz del cáncer de tiroides, especialmente en población de riesgo, podría asociarse a un mejor pronóstico; este hecho debe ponerse en la perspectiva de un adecuado análisis costo efectividad a nivel de estrategia de salud pública para una patología cada vez de mayor prevalencia y de baja mortalidad. A futuro es posible que se planteen terapias quirúrgicas o no quirúrgicas menos invasivas que aseguren que la morbilidad asociada a la terapia no sobrepasen los riesgos de la enfermedad en sí.

\section{Referencias}

1. Schneider A, Ron E. Carcinoma of follicular epithelium. Epidemiology in pathogenesis. En The Thyroid. A Fundamental and clinical text. 9a ed. Editorial Lippincott Williams \& Wilkins, 2005: 889-906.

2. Burgess JR, Tucker P. Incidence trends for papillary thyroid carcinoma and their correlation with thyroid surgery and thyroid fine-needle aspirative citology. Thyroid 2006; 16: 47-53.

3. Cooper DS, Doherty GM, Haugen BR, Kloos RT, Lee SL, Mandel SJ, Mazzaferri EL, Revised American Thyroid Association Management Guidelines for Patients with thyroid Nodules and Differentiated Thyroid Cancer. Thyroid 2009; 19 (11): 1167-218.

4. Leenhardt L, Grosclaude P, Cherie-Challine L. Increased incidence of thyroid carcinoma in France: A true epidemic or thyroid nodule management effects? Report from the ore d thyroid cancer committee. Thyroid 2004; 14 (12): 1056-60.

5. Davies L, Welch $H$. Increasing incidence of thyroid cancer in the United States, 1973-2000. JAMA 2006; 295 (18): 2164-7.

6. Kent WD, Hall SF, Isotalo PA, Houlden RL, George $\mathrm{RL}$, Groome PA. Increased incidence of differentiated thyroid carcinoma and detection of subclinical disease. CMAJ 2007; 177: 1357-61.

7. Burgess JR. Temporal trends for thyroid carcinoma in Australia: an increasing incidence of papillary thyroid carcinoma (1982-1997). Thyroid 2002; 12: 141-9.

8. Fardella C, Jiménez M, González H, León A, Goñi A, 
Cruz F. Características de presentación del microcarcinoma papilar del tiroides. Experiencia retrospectiva de los últimos 12 años. Rev Med Chile 2005; 133: 1305-10.

9. Cerda LJ, Vucetich BN, Mosso GL. Cartas al Editor. Revista Chilena de Endocrinología y Diabetes 2010; 3 (4): 319-20.

10. Campusano CM, Bello FM, González RE, Lam JE, Liberman CG, Munizaga FC, et al. Consenso de diagnóstico y manejo de los nódulos tiroideos no palpables. Rev Med Chile 2004; 132: 1249-55.

11. Sampson Rj, Woolner LB, Bahn RC, Kurland LT. Occult thyroid carcinoma in Olmsted County, Minnesota: prevalence at autopsy compared with that in Hiroshima and Nagasaki, Japan. Cancer 1974; 34: 2072-6.

12. Arellano L, Ibarra A. Occult Carcinoma of the Thyroid Gland Path Res Pract 1984; 179: 92-5.

13. Hegedus L. Clinical practice. The thyroid nodule. N Engl J Med 2004; 351: 1764-71.

14. Muzzo S, Ramírez I, Carvajal F, Biolley E, Leiva L. [Iodine nutrition in school children of four areas of Chile during the year 2001]. Rev Med Chile 2003; 131 (12): 1391-8.
15. Dijkstra B, Prichard RS, Lee A, Kelly LM, Smyth PP, Crotty T, et al. Changing patterns of thyroid carcinoma. Irish J Med Sci 2007; 176: 87-90.

16. Bridget Sinnott, Elaine Ron, and Arthur B. Schneider, 2010. Exposing the Thyroid to Radiation: A Review of Its Current Extent, Risks, and Implications. Endocrine Reviews 2010; 31: 756-773; doi:10.1210/er.2010-0003.

17. Larson SD, Jackson LN, Riall TS, Uchida T, Thomas RP, Qiu S, et al. Increased incidence of well-differentiated thyroid cancer associated with Hashimoto thyroiditis and the role of the PI3k/Akt pathway. J Am Coll Surg 2007; 204: 764-73.

18. Encuesta Nacional de Salud. Chile 2009-2010 http:// www.minsal.gob.cl/portal/url/item/bcb03d7bc28b64dfe040010165012d23.pdf

19. Hay ID, Bergstralh EJ, Goellner JR, Ebersold JR, Grant CS. Predicting outcome in papillary thyroid carcinoma: development of a reliable prognostic scoring system in a cohort of 1779 patients surgically treated at one institution during 1940 through 1989. Surgery 1993; 114 (6): 1050-8. 